\title{
Management of the neurologically deceased organ donor: A Canadian clinical practice guideline
}

\author{
Ian M. Ball MD MSc, Laura Hornby MSc, Bram Rochwerg MD MSc, Matthew J. Weiss MD, Clay Gillrie RN MSN, \\ Michaël Chassé MD PhD, Frederick D’Aragon MD MSc, Maureen O. Meade MD MSc, Karim Soliman MD, \\ Aadil Ali BSc, Samantha Arora MD, John Basmaji MD, J. Gordon Boyd MD PhD, Bernard Cantin MD PhD, \\ Prosanto Chaudhury MD MSc, Marcelo Cypel MD MSc, Darren Freed MD, Anne Julie Frenette PharmD PhD, \\ Pam Hruska RN MSc, Constantine J. Karvellas MD SM, Sean Keenan MD, Andreas Kramer MD, \\ Demetrios James Kutsogiannis MD, Dale Lien MD, Patrick Luke MD, Meagan Mahoney MD, Jeffrey M. Singh MD, \\ Lindsay C. Wilson MHA, Alissa Wright MD MSc, Jeffrey Zaltzman MD MSc, Sam D. Shemie MD
}

Cite as: CMAJ 2020 April 6;192:E361-9. doi: 10.1503/cmaj.190631

D emand for organs continues to exceed their availability, both in Canada and around the world. ${ }^{1}$ Optimizing the medical management of potential organ donors is an important strategy for enhancing organ supply. The explicit goals for management of deceased organ donors in the intensive care unit (ICU) are to stabilize the potential donor and to optimize the number and quality of organs for transplantation.

In 2006, the Canadian Council for Donation and Transplantation produced the first Canadian recommendations for organ donor management, ${ }^{2}$ the result of a 2004 forum - Medical Management to Optimize Donor Organ Potential - in collaboration with the Canadian Critical Care Society, the Canadian Association of Transplantation and the Canadian Society of Transplantation. This forum was the first structured, cooperative assembly of health care professionals in the fields of critical care medicine and transplantation and was a landmark event in Canadian organ donation practice. ${ }^{2}$ The 2006 guideline had an influence on organ donation recommendations internationally; ${ }^{3-6}$ however, it had not been updated to incorporate emerging evidence in organ donor management and critical care medicine and the many advances in guideline development methodology. ${ }^{7}$

The objective of this guideline is to update the existing Canadian recommendations for the medical management of neurologically deceased ("brain-dead") adult and pediatric potential donors for the purposes of single- or multi-organ recovery for transplantation. (The full guideline is available in Appendix 1, at www.cmaj.ca/lookup/suppl/doi:10.1503/cmaj.190631/-/DC1.)

\section{Scope}

The scope of this guideline is the management of the potential organ donor, beginning at the time of the neurologic determination of death and ending at the time of transfer to the surgical recovery team. This includes all aspects of medical care in the ICU for adult

\section{KEY POINTS}

- The objective of this guideline is to update the existing Canadian recommendations for the medical management of neurologically deceased ("brain-dead") adult and pediatric potential donors for the purposes of single- or multi-organ recovery for transplantation

- We suggest against routine administration of thyroid hormone in neurologically deceased potential donors.

- We suggest against routine coronary angiography in neurologically deceased potential donors being assessed for heart donation.

- We suggest maintaining the body temperature of neurologically deceased potential donors in the range of $34^{\circ} \mathrm{C}$ to $35^{\circ} \mathrm{C}$, unless kidneys will not be used for transplantation.

- We recommend a lung-protective ventilation strategy consisting of low tidal volumes $(6-8 \mathrm{~mL} / \mathrm{kg})$, high positive end-expiratory pressure (at least $8 \mathrm{~cm} \mathrm{H}_{2} 0$ ) and recruitment manoeuvres performed after ventilator disconnections in neurologically deceased potential lung donors.

and pediatric organ donors: monitoring, investigations, somatic support, preventive care, drug administration and technical procedures.

Aspects of deceased donation that are not in the scope of this guideline include ICU interventions that precede death by neurologic criteria, the neurologic determination of death, donation after a circulatory determination of death, donation after medical assistance in dying, organ allocation procedures, anesthetic and surgical procedures related to organ recovery or transplantation, and management of the organ recipient.

The clinical practice and ethics of starting donor management interventions before neurologic determination of death are not addressed by this guideline.

The target users of this guideline include organ donation organizations, policy-makers, administrators and clinicians who manage neurologically deceased organ donors. 


\section{Recommendations}

This clinical practice guideline is intended for use in the care of both neurologically deceased adult and pediatric potential organ donors. We based our recommendations on organ donor management literature from both age groups, although we discovered very little pediatric-specific literature. All recommendations apply to pediatric and adult donors, except for the minimal acceptable blood pressure, which should be age adjusted in pediatric patients.

We make 1 strong recommendation, 30 conditional recommendations, 1 "good practice" statement and 4 "no recommendations." Readers will note that the preponderance of donor management literature is limited to lower levels of evidence. The authors acknowledge recent studies (published after this guideline was submitted) in this area, but these do not change the direction and certainty of the recommendations. More information on the evidence supporting each recommendation is available in the full guideline document (Appendix 1).

This synopsis provides information on 4 recommendations that the steering committee deemed likely to have the most impact on clinical practice. A complete summary of all recommendations is available in Table 1.

\section{Thyroid hormone administration}

We suggest against routine thyroid hormone supplementation (conditional recommendation, low-certainty evidence).

We make no recommendation about thyroid hormone supplementation for patients with hemodynamic instability or cardiac dysfunction.

Endogenous catecholamine release is increased during both acute critical illness and neurologic death. Although this causes an increase in systemic vascular resistance, cardiac output is compromised by neurologic death-induced myocardial suppression and a decrease in thyroid hormone release owing to pituitary failure. ${ }^{8-10}$ This state has the potential to compromise transplantation of the heart itself and other organs as a result of impaired perfusion. There is therefore a theoretical advantage to exogenous thyroid hormone supplementation.

Four randomized controlled trials (RCTs) ${ }^{11-14}$ assessed the effect of thyroid hormone on donor hearts and showed no improvement in the number of hearts eligible for transplantation. These studies were included in a 2012 systematic review, ${ }^{15}$ which concluded there was no benefit from any form of thyroid hormone for neurologically deceased potential donors. We excluded 3 other RCTs included in the 2012 systematic review from our analysis as they did not report on any of our outcomes of interest (e.g., reported only on biochemical markers). All of these RCTs included several co-interventions administered to both the intervention and control groups.

In addition to the RCTs, we identified 5 observational studies ${ }^{16-20}$ that reported conflicting results regarding the effect of treatment with thyroid hormone on the number of transplanted hearts. In the largest cohort study of 63593 patients, ${ }^{21}$ patients who received thy- roid hormone had a $9.23 \%$ higher rate of heart recovery. Two additional cohort studies described a multivariate model that included thyroid hormone as a covariable associated with an increased chance of heart transplant eligibility. ${ }^{22,23}$ Thus, for our primary outcome of interest - number of hearts transplanted - the evidence suggests that thyroid hormone is of little benefit, although some larger cohort data suggest increased cardiac recovery.

The guideline panel did consider other outcomes, including cardiac function prerecovery, total number of organs recovered, graft survival and patient survival. Only cardiac function prerecovery was assessed in RCTs and no significant improvement was observed..$^{11-13}$ Cohort data, primarily from the large United Network for Organ Sharing report, ${ }^{21}$ suggest that thyroid hormone replacement is associated with increased cardiac graft and patient survival at 1 and 12 months, and increased number of organs recovered. Although these findings were statistically significant, their interpretation is complicated by the fact that most patients received thyroid replacement as part of a hormone therapy bundle and the effect of thyroid alone is difficult to assess.

Given the absence of established benefit in RCTs comparing thyroid hormone to no thyroid hormone, and the conflicting observational evidence, we suggest against the routine use of thyroid hormone in all potential donors.

We acknowledge that it is accepted practice in many centres to administer thyroid hormone to patients with cardiac dysfunction and that a recommendation against doing so in this subgroup would have added acceptability and feasibility issues. We also acknowledge that there is no published evidence of physiologic harm associated with the administration of thyroid hormone in this context. Given the absence of comparative evidence (RCTs or observational) in this higher-risk population and the potentially substantial acceptability issues, the committee felt the benefits and harms were very closely balanced. As such, we make no recommendation in the subgroup of patients with hemodynamic instability or cardiac dysfunction.

\section{Coronary angiography}

We suggest against routine coronary angiography (conditional recommendation, very low-certainty evidence).

Coronary angiography should be performed in the presence of risk factors for coronary artery disease as determined according to local criteria (good practice statement).

The evidence addressing the benefits of coronary angiography in potential heart donors is of very low quality. There is only 1 study that directly addresses this question. In this retrospective cohort study, Hauptman and colleagues ${ }^{24}$ compared the rates of early graft failure in recipients who received a heart from a donor who underwent coronary angiography versus those who received hearts from donors who did not undergo coronary angiogram. Recipients who received a heart from donors who had an angiogram were less likely to have early graft failure. ${ }^{24}$ No adverse effects were reported. This was a retrospective, nonrandomized study with low internal validity. 


\section{Cardiovascular performance, monitoring and hemodynamic support}

Vasoactive medications

- We suggest intravenous vasopressin as a first-line vasoactive agent for hypotension (conditional recommendation, very low-certainty evidence).

- We suggest intravenous norepinephrine as a second-line agent for hypotension not responding to vasopressin alone (conditional recommendation, very low-certainty evidence).

- We suggest against the use of dopamine at any dose (conditional recommendation, low-certainty evidence).

- We make no recommendation regarding other vasoactive medications and inotropes.

Antihypertensive medications

- We suggest using short-acting intravenous antihypertensive agents as per standard ICU practice to prevent end-organ damage (conditional recommendation, very low-certainty evidence).

Minimal acceptable blood pressure target

- We suggest maintaining mean arterial pressure $\geq 65 \mathrm{~mm} \mathrm{Hg}$ rather than a lower threshold (conditional recommendation, very low-certainty evidence).

Fluid resuscitation

- We suggest infusing crystalloids, rather than colloids, for plasma-volume expansion (conditional recommendation, low-certainty evidence).

Protocolized fluid management

- We make no recommendation regarding the use of fluid management protocols.

\section{Glycemic control and nutrition}

Glucose control

- We suggest maintaining serum glucose levels in the range of 6-10 mmol/L, rather than a lower range of 4-6 mmol/L (conditional recommendation, very low-certainty evidence).

Glucose, insulin and potassium administration

- We suggest against the infusion of combined solutions of glucose, insulin and potassium (conditional recommendation, very low-certainty evidence).

Nutritional support

- We suggest providing enteral nutrition as compared with no nutritional support (conditional recommendation, very low-certainty evidence)

\section{Diabetes insipidus and hypernatremia}

Serum sodium control

- We suggest maintaining serum sodium concentration in the normal range (135-155 mmol/L) (conditional recommendation, very low-certainty evidence).

Diabetes insipidus and vasopressin

- We suggest treating diabetes insipidus with desmopressin or vasopressin during hemodynamic stability (conditional recommendation, very low-certainty evidence).

- We suggest treating diabetes insipidus with vasopressin during hemodynamic instability (conditional recommendation, very low-certainty evidence).

\section{Hormonal therapy}

Thyroid hormone

- We suggest against routine thyroid hormone supplementation (conditional recommendation, low-certainty evidence).

- We make no recommendation about thyroid hormone supplementation for hemodynamic instability or cardiac dysfunction.

Corticosteroids

- We suggest intravenous corticosteroid therapy for donors requiring vasopressor support (conditional recommendation, low-certainty evidence).

- We make no recommendation about high-dose corticosteroid therapy for potential lung donors.

\section{Transfusion therapy}

Transfusion threshold

- We suggest withholding red blood cell transfusions unless hemoglobin levels fall below $70 \mathrm{~g} / \mathrm{L}$ (conditional recommendation, very low certainty in evidence).

Correction of coagulopathy and thrombocytopenia

- We suggest that in the absence of clinically significant bleeding, transfusions of fresh frozen plasma be withheld altogether, and that platelet transfusions be withheld unless platelet levels fall below $10 \times 10^{9} / \mathrm{L}$ (conditional recommendation, very low certainty in evidence). 
Table 1 (part 2 of 2): Summary of recommendations

\section{Bacterial infections}

Antibiotics

- We suggest reserving antibiotic therapy for the treatment of known or suspected infection (conditional recommendation, very low-certainty evidence).

Routine cultures

- We suggest that screening cultures of blood, urine and sputum be performed at intervals consistent with general ICU practice and patient clinical status (conditional recommendation, very low-certainty evidence).

\section{Organ-specific considerations: heart, lungs and intra-abdominal organs}

Cardiac assessment tools

- We suggest against routine use of pulmonary artery catheters (conditional recommendation, very low-certainty evidence).

- We suggest serial echocardiography at intervals consistent with general ICU practice (conditional recommendation, very low-certainty evidence).

\section{Cardiac biomarkers}

- We suggest against the measurement of serum cardiac biomarkers (conditional recommendation, very low-certainty evidence).

Coronary angiography

- We suggest against routine coronary angiography (conditional recommendation, very low-certainty evidence).

- Coronary angiography should be performed in the presence of risk factors for coronary artery disease as determined according to local criteria (good practice statement).

Lung-protective ventilation

- We recommend a lung-protective ventilation strategy consisting of low tidal volumes $(6-8 \mathrm{~mL} / \mathrm{kg})$, high positive end-expiratory pressure (at least $8 \mathrm{~cm} \mathrm{H}_{2} \mathrm{O}$ ) and recruitment manoeuvres after ventilator disconnections in potential lung donors (strong recommendation, moderatecertainty evidence).

Bronchoscopy

- We suggest diagnostic bronchoscopy be performed for potential lung donors (conditional recommendation, low-certainty evidence).

Inhaled $\beta$-agonist therapy

- We suggest against routine use of inhaled $\beta$-agonists (conditional recommendation, moderate-certainty evidence).

Chest radiography and computed tomography scan

- We suggest a single routine diagnostic chest radiograph for lung donors and additional chest imaging as clinically indicated (conditional recommendation, low-certainty evidence).

Albumin: creatinine ratio screening

- We suggest using the albumin:creatinine ratio for detecting microalbuminuria when assessing potential kidney donors with type 1 or 2 diabetes mellitus (conditional recommendation, very low certainty in evidence).

Hemoglobin $\mathrm{HbA}_{1 c}$ testing

- We suggest that hemoglobin $\mathrm{HbA}_{1 c}$ testing be performed in potential donors being considered for pancreas donation (conditional recommendation, very low-certainty evidence)

Abdominal imaging

- We suggest that abdominal CT or ultrasound should be used only in those with age $>50 \mathrm{yr}$, comorbid conditions, high body mass index, or clinical history of malignancy (conditional recommendation, low-certainty evidence).

\section{Other therapeutic interventions}

Therapeutic hypothermia

- We suggest maintaining the core body temperature in the range of $34^{\circ} \mathrm{C}-35^{\circ} \mathrm{C}$, unless kidneys will not be used for transplantation, in which case normothermia is appropriate (conditional recommendation, low-certainty evidence).

Duration of donor management

- For potential donors with acute organ injury, we make no recommendation regarding timing of organ recovery surgery or optimal duration of ICU donor management (conditional recommendation, very low-certainty evidence).

Note: $\mathrm{CT}$ = computed tomography, $\mathrm{HbA}_{1 \mathrm{c}}=$ glycosylated hemoglobin, $\mathrm{ICU}=$ intensive care unit .

We also considered the logistics of coronary angiography. Coronary angiography requires patient transport outside of the ICU, exposes the donor organs to potentially harmful intraarterial contrast, is not available in all centres where patients who are potential donors are admitted, and is relatively costly and resource intensive.

Considering the limited evidence of benefit and the risks described above, we suggest against using routine coronary 
angiography. As a good practice statement, however, we suggest that coronary angiography be considered in donors with risk factors for coronary artery disease because despite the lack of supporting evidence for angiography in this clinical situation, it is expected that the benefit of avoiding transplant of a heart with coronary artery disease will outweigh the risk related to logistics and intra-arterial contrast.

\section{Lung-protective ventilation}

We recommend a lung-protective ventilation strategy consisting of low tidal volumes $(6-8 \mathrm{~mL} / \mathrm{kg})$, high positive end-expiratory pressure (PEEP) (at least $8 \mathrm{~cm} \mathrm{H}_{2} \mathrm{O}$ ) and recruitment manoeuvres after ventilator disconnections in potential lung donors (strong recommendation, moderate-certainty evidence).

Ventilation with low tidal volumes is the standard of care for many ICU patient populations and is supported by experimental evidence and physiologic rationale. Some of the highest-quality evidence in organ donor management is in the area of donor ventilation.

We defined a lung-protective ventilatory strategy as low tidal volumes, high PEEP and recruitment manoeuvres. We were unable to identify literature that evaluated any of these 3 components in isolation. One RCT and 3 observational studies examined mechanical ventilation with a lung-protective approach in neurologically deceased potential donors. ${ }^{25-28}$ The RCT compared a lung-protective approach (tidal volumes of $6-8 \mathrm{~mL} / \mathrm{kg}$ predicted body weight, PEEP $8-10 \mathrm{~cm} \mathrm{H}_{2} \mathrm{O}$, recruitment manoeuvres after disconnections from the ventilator (and an additional protocol for apnea testing) to conventional ventilation (tidal volumes $>8 \mathrm{~mL} / \mathrm{kg}$ predicted body weight, $\mathrm{PEEP}<8 \mathrm{~cm} \mathrm{H}_{2} \mathrm{O}$, no protocolized recruitment manoeuvres and apnea tests off the ventilator). ${ }^{25}$ The protective strategy was continued for a minimum of 6 hours, and potential donors assigned to this strategy were twice as likely to proceed to lung procurement. There was no signal of harm among the lung recipients; nor was there any signal of harm from the protective ventilation strategy with respect to the recovery of other organs.

Three observational studies with historical control groups evaluated the effect of a comprehensive lung-donor management strategy. ${ }^{26-28}$ Two of these studies involved the ventilation protocol tested in the RCT. ${ }^{26,27}$ The third study involved higher PEEP of $15 \mathrm{~cm} \mathrm{H} \mathrm{H}_{2} \mathrm{O}$, without the other components of lungprotective ventilation. All 3 studies showed an improved lung procurement rate with implementation of their comprehensive lung donor management strategies, and 1 of the 3 studies showed increased recipient survival. ${ }^{28}$

The committee considered potential drawbacks not explored in these studies. First, as was the case in all the studies, the criteria for lung donation eligibility frequently involves the ratio of arterial oxygen partial pressure to fractional inspired oxygen $\left(\mathrm{PaO}_{2}: \mathrm{FiO}_{2}\right.$ ratio) as a measure of lung function. However, PEEP is a therapeutic intervention that may transiently improve the $\mathrm{PaO}_{2}: \mathrm{FiO}_{2}$ ratio in some physiologic states. ${ }^{29-31}$ Thus, there is a risk that high PEEP ventilation allows relatively suboptimally functioning lungs to appear numerically viable for transplant at the time of eligibility assessment. In the RCT, lung-protective ventilation was not associated with an increase in criterion-eligible lungs being declined based on gross inspection or other surgical opinion. ${ }^{25}$

Second, increasing mean airway pressure via increased PEEP has the potential to decrease cardiac output. ${ }^{32}$ It is possible that the decreased cardiac output outweighs any benefit on graft oxygen delivery derived from improved arterial oxygenation. In the donation population, this may be best assessed through the viability of other organs in potential donors managed with lungprotective ventilation, but this outcome was not reported in the included studies.

Given that the evidence for a benefit from lung-protective ventilation is greatest for lung donation, we considered narrowing our recommendations to potential lung donors only. However, the candidacy of specific organs for donation is often not known in the early stages of donor care, and a delay in initiating lung-protective ventilation will reduce its benefit. We therefore recommend that lung-protective ventilation be started as soon as possible in all potential neurologically deceased donors. Intensivists and respiratory therapists are experienced with lungprotective ventilation in the critically ill, and this approach does not incur any additional costs with respect to equipment or human resources. We therefore do not expect major barriers to implementing this recommendation routinely in neurologically deceased donors.

\section{Therapeutic hypothermia}

We suggest maintaining the core body temperature in the range of $34-35^{\circ} \mathrm{C}$, unless kidneys will not be used for transplantation, in which case normothermia is appropriate (conditional recommendation, low-certainty evidence).

In the single RCT in this area, ${ }^{33}$ the induction of mild therapeutic hypothermia $\left(34^{\circ} \mathrm{C}-35^{\circ} \mathrm{C}\right)$ in donors was shown to reduce the occurrence of delayed graft function in kidneys, a benefit that was even more substantial in the extended criteria donor population. There was no evidence of harm in the intervention group and the recovery of other organs was not affected in the hypothermia group. Graft outcome of other transplanted organs was not reported.

A retrospective analysis of mild spontaneous hypothermia $\left(<36^{\circ} \mathrm{C}\right)$ was performed from a data set designed to study dopamine in the organ donor population. ${ }^{34}$ Similarly to the RCT described above, the study showed a reduction in kidney delayed graft function in the hypothermia group. There was no difference in 5-year graft survival. The same data set used to study cardiac donors identified a lower 3-year graft survival in donors with spontaneous temperature less than $36.2^{\circ} \mathrm{C} .{ }^{35}$ Given the methodological limitations of studying spontaneous hypothermia, causation versus association for outcomes was an issue; evidence was downgraded to low.

Targeted temperature management is now common practice in most critical care units. Although there are only 3 studies evaluating this intervention in organ donors, the absence of any identified harm, combined with the fact that the most commonly 
recovered organ is the kidney, led our guideline committee to suggest mild hypothermia in all organ donors until kidneys have been ruled out for donation.

We acknowledge that there are minimal data on the effect of mild hypothermia on nonrenal organs and that the evidence for cardiac donors is weak. New evidence could change this recommendation. The considerations for hypothermia are stronger when only kidneys are considered in an extended criteria donor.

\section{Methods}

\section{Guideline panel composition}

Canadian Blood Services assembled a 7-member guideline steering committee consisting of a critical care physician with expertise in Grading of Recommendations Assessments, Development and Evaluation (GRADE; www.gradeworkinggroup.org/) methodology (B.R.), a policy-maker (L.H.), 3 content experts on deceased donation (M.W., S.S., I.B.), and 2 project managers (C.G., L.W.).

The 23-member guideline panel selected by the steering committee included representation from critical care medicine (adult and pediatric), critical care nursing and pharmacy; an infectious disease specialist; organ donation specialist physicians; and people with organ-specific expertise in transplant cardiology, cardiac surgery, pulmonology, lung surgery, urology, nephrology and abdominal surgery. We included 2 patient partners, a family member of a deceased multi-organ donor and a heart transplant recipient. Other panel members included representatives from specific knowledge user groups: the Canadian Critical Care Society, the Canadian Society of Transplantation, the Canadian Association of Critical Care Nurses and the Canadian Donation and Transplantation Research Program. By design, the guideline panel included geographic diversity from 4 provinces (Ontario, Quebec, Alberta and $\mathrm{BC}$ ), representing the most active donation and transplant programs in Canada. The full participant list and description of the guideline methodology is available in Appendix 1.

\section{Guideline development}

We reviewed the results of a systematic review of published organ donor management guidelines ${ }^{36}$ before deciding on the scope of our effort. This review of 27 guidelines from 22 countries provided an overview of topics that had been covered by other groups and informed our list of 29 potential PICO (Patient, Intervention, Comparator, Outcome) questions (Appendix 1).

The guideline panel was divided into 3 smaller working groups that selected outcomes of interest for each question. The overall group explicitly rated these outcomes for their relative importance from "not important" to "critical" according to GRADE guidance. ${ }^{37}$ In cases of disagreement, decisions were made by group discussion and consensus. We developed a hierarchy of outcomes to inform these discussions: clinical optimization during the interval between death declaration and organ recovery; increased organ acceptance, recovery and transplantation; an increase in organ recovery; improved graft function; graft survival; and recipient survival and quality of life.

Within the 3 working groups, panel members were assigned PICO questions in pairs and collaborated with information technologists or medical librarians to design and conduct search strategies for each of the 29 PICO questions. Our population of interest was neurologically deceased organ donors. Primary searches were limited to human studies reported in English, either in full text or abstract. At minimum for each question, we searched MEDLINE (PubMed) and the Cochrane Central Register of Controlled Trials (CENTRAL). Primary searches were restricted to human studies reported in English, from 1981 to present, either in full text or abstract (see Appendix 1 for details of the search strategies, including individual end dates). Two panel members independently screened all relevant citations of each PICO question for eligibility. Disagreements were resolved through consensus and discussion. Searches were completed by September 2018. Steering committee members monitored the literature up until January 24, 2020, for recent publications that could affect the direction or certainty of any recommendations.

In situations where little to no direct research evidence was available in the organ donation or transplantation population, we extended our search to indirect evidence. In most cases, this referred to studies in the general ICU population, but in some cases, it included animal studies or trials in other patient populations.

In their assigned pairs, panel members abstracted study characteristics and outcome data from all eligible studies for each PICO question. In addition to clinical data, we also assessed individual study risk of bias using the Cochrane Risk of Bias tool ${ }^{38}$ for RCTs and the Newcastle-Ottawa tool ${ }^{39}$ for observational studies. When possible, we pooled outcome data for each PICO question and performed meta-analyses using the Cochrane Collaboration Review Manager 5.2. ${ }^{40}$ Once this analysis was completed, we assessed the overall certainty (confidence) in the effect estimate for each outcome of interest following the GRADE approach ${ }^{41}$ based on these criteria: risk of bias, precision, consistency, directness of the evidence, risk of publication bias, presence of a dose-effect relationship, magnitude of effect and an assessment of the effect of plausible residual confounding or bias. Considering all of these elements, the strength of evidence for each outcome was categorized as high, moderate, low or very low. Steering committee members with GRADE expertise and experience (B.R. and L.H.) oversaw this process.

Pairs of panel members prepared evidence summaries for each question in collaboration with the methodologist (B.R.), following the GRADE approach ${ }^{42}$ and using the GRADEpro Guideline Development Tool online software. ${ }^{43}$

Based on these evidence summaries, the 3 working groups developed recommendations for each PICO question. We used the GRADE Evidence to Decision framework to facilitate discussion on each recommendation. ${ }^{44}$ Recommendation development was based on the certainty of the evidence, the balance of desirable and undesirable consequences of compared management options, assumptions about the values and preferences associated with the decision, implications for resource use and health equity, the acceptability of an intervention to stakeholders, and feasibility of implementation. The guideline panel assessed each recommendation for pediatricspecific considerations. The 3 working groups presented their recommendations to the entire guideline panel for further review and discussion. Disagreements were resolved by consensus. 
Table 2: Interpretation of strong and weak recommendations for different stakeholders

\begin{tabular}{|c|c|c|}
\hline Implications & Strong recommendation & Conditional (weak) recommendation \\
\hline For patients & $\begin{array}{l}\text { Most individuals in this situation would want the } \\
\text { recommended course of action and only a small } \\
\text { proportion would not. }\end{array}$ & $\begin{array}{l}\text { The majority of individuals in this situation, if fully } \\
\text { informed, would choose the suggested course of action, } \\
\text { but some would not. }\end{array}$ \\
\hline For clinicians & $\begin{array}{l}\text { Most individuals should receive the intervention. } \\
\text { Adherence to this recommendation according to the } \\
\text { guideline could be used as a quality criterion or } \\
\text { performance indicator. }\end{array}$ & $\begin{array}{l}\text { The care needs of individual donors may vary as a result of } \\
\text { comorbidities, and the practice of individual clinicians may } \\
\text { vary in these circumstances, largely owing to the lack of } \\
\text { evidence to address these situations. }\end{array}$ \\
\hline For policy-makers & $\begin{array}{l}\text { The recommendation can be adapted as policy in most } \\
\text { situations }\end{array}$ & $\begin{array}{l}\text { Policy-making will require substantial debate and } \\
\text { involvement of various stakeholders. }\end{array}$ \\
\hline
\end{tabular}

In accordance with the GRADE approach, ${ }^{45}$ we used the phrasing "we recommend" for strong recommendations and "we suggest" for conditional recommendations. Table 2 provides a guide to interpretation of these recommendations for intended stakeholders, including patients, clinicians and health policy-makers.

The guideline panel endeavoured to provide recommendations for each original PICO question. In the exceptional circumstance where there was insufficient evidence for or against an intervention, or if there was a perceived balance between net benefits and net harms, then the panel made "no recommendation." "Good practice statements" were made when there was a high level of certainty about the effect of the therapy, but little direct supporting evidence. ${ }^{47}$

\section{External review}

Representatives of the supporting organizations, including the Canadian Critical Care Society, the Canadian Society of Transplantation and the Canadian Association of Critical Care Nurses, performed external reviews. The lead author (I.B.) incorporated the feedback into the guideline and steering committee members edited it. The entire guideline panel had an opportunity to review the final version of the guideline.

\section{Management of competing interests}

All guideline panel members signed a confidentiality agreement at the inaugural meeting in 2017, where they were also asked to disclose any potential direct and indirect competing interests using a Canadian Blood Services competing interests documentation form. These were collected again a year later and before publication. Canadian Blood Services personnel and the steering committee reviewed the declarations. Several panel members have professional roles in organ donation administration, with governmental not-for-profit entities, or have funded scientific research; however, no steering committee or panel member was judged to have a relevant direct competing interest.

\section{Implementation}

No specific dosing regimens were recommended for either pediatric or adult patients, and groups planning to use these recom- mendations to create bedside treatment protocols or standardized order sets should consult with critical care pharmacists with experience treating the target population.

Members of our guideline panel have created "evidence bulletins" (1-page, laminated cards explaining key recommendations) for distribution to ICUs in partnership with interested provincial organ donation organizations, and leaders in ICU education (bit. ly/dmevidencebulletins). We have also developed adult and pediatric order sets for use by provincial organ donation organizations and the hospitals that they service (Appendix 1), as well as a family brochure (bit.ly/dmfamilybrochure).

In some cases, a PICO question addressed whether or not to administer a particular medication in a particular circumstance. We made evidence-based recommendations about the appropriateness of the treatment but did not necessarily survey the literature for the best medication or dose from its class (e.g., corticosteroids). In these cases, the panel identified (but did not formally recommend) commonly prescribed medications and their usual doses, in order to help clinicians at the bedside.

Canadian Blood Services personnel regularly monitor the literature and an update is triggered when there have been any of the following:

- New evidence on the existing benefits and harms of interventions;

- Changes in outcomes considered important;

- Changes in available interventions;

- Changes in evidence that current practice is optimal;

- Changes in values placed on outcomes; or

- Changes in resources available for health care.

\section{Other guidelines}

Members of this group performed a systematic review of published organ donor management guidelines ${ }^{36}$ to guide the scope of this guideline document. This review of 27 guidelines from 22 countries provided an overview of topics that had been covered by other groups and informed our initial list of potential PICO questions. In contrast to previous guidelines on organ donor management, this guideline was derived using GRADE methodology. 


\section{Gaps in knowledge}

An important limitation of these guidelines is that organ donor management research is predominantly observational in design. Many important clinical questions have not been addressed by RCTs. In addition, our guideline committee identified more PICO questions than we were able to process in this iteration. A list of items for generation of potential PICO questions and consideration for future guidelines is included in the full guidance document (Appendix 1).

\section{Conclusion}

The multidisciplinary guideline panel reviewed the literature for 29 PICO questions and generated 1 strong recommendation, 33 conditional recommendations, and 1 good practice statement as part of a comprehensive and evidence-based clinical practice guideline for organ donor management. It is our hope that this work can optimize use of the scarce resource that donated organs continue to be.

\section{References}

1. Organ donation and transplantation in Canada: system progress report - 2017 update. Ottawa: Canadian Blood Services; 2018. Available: https://blood.ca/sites/ default/files/System_Progress_Report_2017_Update_FINAL-EN.pdf (accessed 2019 Feb. 10).

2. Shemie SD, Ross H, Pagliarello J, et al. Organ donor management in Canada: recommendations of the forum on medical management to optimize donor organ potential. CMAJ 2006;174:S13-32.

3. Kotloff RM, Blosser S, Fulda GJ, et al. Management of the potential organ donor in the ICU: Society of Critical Care Medicine/American College of Chest Physicians/ Association of Organ Procurement Organizations Consensus Statement. Crit Care Med 2015;43:1291-325.

4. Ellis MKM, Sally MB, Malinoski DJ. Management of the potential organ donor. In: Salim A, Brown C, Inaba K, et al., editors. Surgical critical care therapy: a clinically oriented practical approach. Cham (Switzerland): Springer International Publishing; 2018:67-75.

5. Pandit RA, Zirpe KG, Gurav SK, et al. Management of potential organ donor: Indian Society of Critical Care Medicine: position statement. Indian J Crit Care Med 2017;21:303-16.

6. Grissom TE, Richards JE, Herr DL. Critical Care management of the potential organ donor. Int Anesthesiol Clin 2017;55:18-41.

7. Schünemann HJ, Wiercioch W, Etxeandia I, et al. Guidelines 2.0: systematic development of a comprehensive checklist for a successful guideline enterprise. CMAJ 2014;186:E123-42.

8. Novitzky D, Cooper DK, Rosendale JD, et al. Hormonal therapy of the brain-dead organ donor: experimental and clinical studies. Transplantation 2006;82:1396-401.

9. Powner DJ, Hendrich A, Lagler RG, et al. Hormonal changes in brain dead patients. Crit Care Med 1990;18:702-8.

10. Cooper LB, Milano CA, Williams M, et al. Thyroid hormone use during cardiac transplant organ procurement. Clin Transplant 2016;30:1578-83.

11. Jeevanandam V. Triiodothyronine: spectrum of use in heart transplantation. Thyroid 1997; 7:139-45.

12. Mariot J, Jacob F, Voltz C, et al. Value of hormonal treatment with triiodothyronine and cortisone in brain dead patients [article in French]. Ann Fr Anesth Reanim 1991;10:321-8.

13. Randell TT, Hockerstedt KA. Triiodothyronine treatment in brain-dead multiorgan donors - a controlled study. Transplantation 1992;54:736-8.

14. Venkateswaran RV, Patchell VB, Wilson IC, et al. Early donor management increases the retrieval rate of lungs for transplantation. Ann Thorac Surg 2008;85:278-86, discussion 86.

15. Macdonald PS, Aneman A, Bhonagiri D, et al. A systematic review and metaanalysis of clinical trials of thyroid hormone administration to brain dead potential organ donors. Crit Care Med 2012;40:1635-44.

16. Nath DS, Ilias Basha $\mathrm{H}$, Liu MH, et al. Increased recovery of thoracic organs after hormonal resuscitation therapy. J Heart Lung Transplant 2010;29:594-6.

17. Novitzky D, Cooper DK, Reichart B. Hemodynamic and metabolic responses to hormonal therapy in brain-dead potential organ donors. Transplantation 1987;43:852-4.
18. Orlowski JP, Spees EK. Improved cardiac transplant survival with thyroxine treatment of hemodynamically unstable donors: $95.2 \%$ graft survival at 6 and 30 months. Transplant Proc 1993;25(1 pt 2):1535.

19. Roels L, Pirenne J, Delooz H, et al. Effect of triiodothyronine replacement therapy on maintenance characteristics and organ availability in hemodynamically unstable donors. Transplant Proc 2000;32:1564-6.

20. Salim A, Martin M, Brown C, et al. Using thyroid hormone in brain-dead donors to maximize the number of organs available for transplantation. Clin Transplant 2007;21:405-9.

21. Novitzky D, Mi Z, Collins JF, et al. Increased procurement of thoracic donor organs after thyroid hormone therapy. Semin Thorac Cardiovasc Surg 2015;27:123-32.

22. Rosendale JD, Kauffman HM, McBride MA, et al. Hormonal resuscitation yields more transplanted hearts, with improved early function. Transplantation 2003;75:1336-41.

23. Malinoski DJ, Daly MC, Patel MS, et al. Achieving donor management goals before deceased donor procurement is associated with more organs transplanted per donor. J Trauma 2011;71:990-5, discussion 6 .

24. Hauptman PJ, O'Connor KJ, Wolf RE, et al. Angiography of potential cardiac donors. J Am Coll Cardiol 2001;37:1252-8.

25. Mascia L, Pasero D, Slutsky AS, et al. Effect of a lung protective strategy for organ donors on eligibility and availability of lungs for transplantation: a randomized controlled trial. JAMA 2010;304:2620-7.

26. Miñambres E, Coll E, Duerto J, et al. Effect of an intensive lung donor-management protocol on lung transplantation outcomes. J Heart Lung Transplant 2014;33:178-84.

27. Miñambres E, Perez-Villares JM, Chico-Fernandez M, et al. Lung donor treatment protocol in brain dead-donors: a multicenter study. J Heart Lung Transplant 2015;34:773-80.

28. Angel LF, Levine DJ, Restrepo MI, et al. Impact of a lung transplantation donor management protocol on lung donation and recipient outcomes. Am J Respir Crit Care Med 2006;174:710-6.

29. Villar J, Perez-Mendez L, Lopez J, et al. An early PEEP/FIO2 trial identifies different degrees of lung injury in patients with acute respiratory distress syndrome. Am J Respir Crit Care Med 2007;176:795-804.

30. Ferguson ND, Kacmarek RM, Chiche JD, et al. Screening of ARDS patients using standardized ventilator settings: influence on enrollment in a clinical trial. Intensive Care Med 2004;30:1111-6.

31. Estenssoro E, Dubin A, Laffaire E, et al. Impact of positive end-expiratory pressure on the definition of acute respiratory distress syndrome. Intensive Care Med 2003;29:1936-42.

32. Dorinsky PM, Whitcomb ME. The effect of PEEP on cardiac output. Chest 1983;84:210-6.

33. Niemann CU, Feiner J, Swain S, et al. Therapeutic hypothermia in deceased organ donors and kidney-graft function. N Engl J Med 2015;373:405-14.

34. Schnuelle P, Mundt HM, Druschler F, et al. Impact of spontaneous donor hypothermia on graft outcomes after kidney transplantation. Am J Transplant 2018;18:704-14.

35. Schnuelle P, Benck U, Kramer BK, et al. Impact of donor core body temperature on graft survival after heart transplantation. Transplantation 2018;102:1891-900.

36. Frenette AJ, Williamson D, Rochwerg B, et al. Worldwide management of brain dead organ donor: a systematic review of guidelines [poster A742018]. International Symposium on Intensive Care and Emergency Medicine; 2018 Mar. 20-23; Brussels.

37. Guyatt GH, Oxman AD, Kunz R, et al. GRADE guidelines: 2. Framing the question and deciding on important outcomes. J Clin Epidemiol 2011;64:395-400.

38. Higgins JP, Altman DG, Gotzsche PC, et al. The Cochrane Collaboration's tool for assessing risk of bias in randomised trials. BMJ 2011;343:d5928.

39. Sanderson S, Tatt ID, Higgins JP. Tools for assessing quality and susceptibility to bias in observational studies in epidemiology: a systematic review and annotated bibliography. Int J Epidemiol 2007;36:666-76.

40. Review Manager (RevMan). 5.2 ed. Copenhagen: Cochrane Collaboration; 2012.

41. Balshem $\mathrm{H}$, Helfand $\mathrm{M}$, Schünemann $\mathrm{HJ}$, et al. GRADE guidelines: 3 . Rating the quality of evidence. J Clin Epidemiol 2011;64:401-6.

42. Guyatt $\mathrm{G}$, Oxman AD, Akl EA, et al. GRADE guidelines: 1. Introduction-GRADE evidence profiles and summary of findings tables. J Clin Epidemiol 2011;64:383-94.

43. Guideline Development Tool. Hamilton: McMaster University and Evidence Prime; 2015. Available: www.guidelinedevelopment.org (accessed 2017 Apr. 1).

44. Schünemann HJ, Mustafa R, Brozek J, et al. GRADE guidelines: 16. GRADE evidence to decision frameworks for tests in clinical practice and public health. $J$ Clin Epidemiol 2016;76:89-98.

45. Andrews JC, Schünemann HJ, Oxman AD, et al. GRADE guidelines: 15. Going from evidence to recommendation-determinants of a recommendation's direction and strength. J Clin Epidemiol 2013;66:726-35.

46. Guyatt GH, Oxman AD, Kunz R, et al. Going from evidence to recommendations. BMJ 2008;336:1049-51.

47. Tugwell $P$, Knottnerus JA. When does a good practice statement not justify an evidence-based guideline? J Clin Epidemiol 2015;68:477-9. 
Competing interests: John Gordon Boyd reports receiving salary support from the Trillium Gift of Life Network, nonfinancial support from Caster Medical and a grant from Physician Services Incorporated, outside the submitted work. Maureen Meade reports receiving a knowledge translation grant from Canadian Blood Services, and salary support as a hospital donation physician for Trillium Gift of Life Network. Matthew J Weiss reports receiving ongoing funding from Transplant Québec for his work as medical director of organ donation. Karim Soliman reports receiving a stipend to support education in the province from the Trillium Gift of Life Network. Jeffrey Singh reports receiving a salary as the regional medical lead for donation at Trillium Gift of Life Network. Samantha Arora reports receiving a knowledge translation grant from Canadian Blood Services, and salary support for Hospital Donation Physician work with Trillium Gift of Life, outside the submitted work. Ian Ball reports receiving a consultant stipend from Trillium Gift of Life Network for work as a donation physician. Sam Shemie reports receiving salary support as a medical advisor for deceased organ donation, Canadian Blood Services. Laura Hornby reports receiving consulting fees from Canadian Blood Services. Prosanto Chaudhury reports receiving a salary as a medical director of Transplant Québec, and receiving honoraria from Novartis, Ipsen and Amgen for participation in advisory boards and for hosting invited speakers, outside the submitted work. Alissa Wright reports receiving a stipend for work as an Infectious Disease consultant for BC Transplant. Sean Keenan reports receiving ongoing funding for his work as Provincial Medical Director for BC Transplant. No other competing interests were declared.

This article has been peer reviewed.

Affiliations: Departments of Medicine (Ball), Internal Medicine (Basmaji) and Surgery (Luke), Western University, London, Ont.; Children's Hospital of Eastern Ontario Research Institute (Hornby); Deceased Donation (Hornby, Shemie, Wilson), Canadian Blood Services, Ottawa, Ont.; Department of Medicine (Division of Critical Care) and Department of Health Research Methods, Evidence and Impact (Rochwerg, Meade), McMaster University, Hamilton, Ont.; Faculté de médecine (Weiss), Université Laval; Transplant Quebec (Weiss), Québec, Que.; Canadian Blood Services (Gillrie), Ottawa, Ont.; Department of Medicine and School of Public Health (Chassé), University of Montreal, Montréal, Que.; Department of Anesthesiology, Faculty of Medicine and Health Sciences (D'Aragon), University of Sherbrooke, Sherbrooke, Que.; Critical Care (Soliman), Queen's University, Kingston, Ont.; Latner Thoracic Surgery Research Laboratories, Institute of Medical Sciences (Ali), University of Toronto, Toronto, Ont.; Northern Ontario School of Medicine (Arora), Thunder Bay, Ont.; Department of Medicine (Neurology) and Critical Care, Centre for Neuroscience Studies (Boyd), Queen's University, Kingston, Ont.; Department of Medicine (Cantin), Université Laval, Québec, Que.; Gerald Bronfman Department of Oncology (Cantin), McGill University, Montréal, Que.; Departments of Surgery (Cypel) and Medicine (Singh), University of Toronto, Toronto, Ont.; Division of Cardiac Surgery (Freed), University of Alberta, Edmonton, Alta.; Faculty of Pharmacy (Frenette), University of Montreal, Montréal, Que.; Alberta Health Services (Hruska), Calgary, Alta.; Department of Critical Care Medicine (Karvellas), University of Alberta, Edmonton, Alta.; BC Transplant (Keenan), Vancouver, BC; Division of Critical Care (Keenan), University of British Columbia, Vancouver, BC; Departments of Critical Care Medicine and Clinical Neurosciences, Hotchkiss Brain Institute (Kramer), University of Calgary, Calgary, Alta.; Faculty of Medicine and Dentistry (Kutsogiannis), University of Alberta, Edmonton, Alta.; Department of Medicine (Lien), University of Alberta, Edmonton, Alta.; London Health Sciences Centre (Luke), London, Ont.; Department of Pediatrics (Mahoney), University of
Calgary, Calgary, Alta.; Division of Infectious Diseases (Wright), University of British Columbia, Vancouver, BC; Division of Nephrology (Zaltzman), University of Toronto, Toronto, Ont.; Department of Pediatrics (Shemie), McGill University, Montréal, Que.

Contributors: Ian Ball, Laura Hornby, Bram Rochwerg, Matthew Weiss and Sam Shemie conceived the guideline development project. All of the authors contributed to the design of the work, and the acquisition, analysis and interpretation of data. All of the authors drafted the manuscript, revised it critically for important intellectual content, gave final approval of the version to be published and agreed to be accountable for all aspects of the work.

Funding: This project was financially supported by Canadian Blood Services through a contribution from Health Canada in support of developing leading practices. Canadian Blood Services is a national, not-for-profit charitable organization. In the domain of organ and tissue donation and transplantation, it provides national services in the development of leading practices, system performance measurement, interprovincial organ sharing registries, and public awareness and education. Canadian Blood Services is not responsible for the management or funding of any Canadian organ donation organizations or transplant programs. Canadian Blood Services receives its funding from the provincial and territorial Ministries of Health and from the federal government (through Health Canada). The services offered by the Alberta Strategy for Patient-Oriented Research SUPPORT Unit (AbSPORU) Knowledge Translation Platform were provided in kind, with support from their funders Alberta Innovates and the Canadian Institutes of Health Research.

Acknowledgements: The authors especially thank Ms. Kathryn Ivison and Ms. Vanya Lang, the donor family member and patient partner, whose experiential perspectives kept this initiative focused on the overarching purpose: providing high-quality end-of-life care for deceased organ donors and improving outcomes for transplant recipients. The authors acknowledge the information services support they received to design and execute the search strategies for the systematic reviews used to inform this guideline: Thane Chambers, MLIS, Research Impact Librarian, Health Sciences Library, University of Alberta; Liza Chan, MLIS, Research Librarian, John W. Scott Health Sciences Library, University of Alberta and Alberta Innovates; Rachel Couban, MISt, National Pain Centre Research Coordinator, Department of Anesthesiology, McMaster University; Patrice Dupont, MLIS, Librarian, Bibliothèque de la Santé, Université de Montréal; Ashley Farrell, MLIS, Information Specialist, University Health Network; Robin Featherstone, MLIS, Information Specialist and Research Librarian, Alberta Research Centre for Health Evidence, University of Alberta; Elena Guadagno, MLIS, Medical Librarian, McConnell Resource Centre, McGill University Health Centre; Lorraine Toews, MLIS, Librarian, Health Sciences Library, University of Calgary; Daniela Ziegler, MSI, Librarian/Informationist, Direction de l'enseignement et de l'Académie Centre hospitalier de l'Université de Montréal. Additionally, the authors acknowledge Thamer Alaifan, Department of Medicine, Critical Care, McMaster University, for his work on the development of the adult and pediatric standing orders. The authors also acknowledge Dr. Darren Malinoski, Division of Trauma, Critical Care and Acute Care Surgery, School of Medicine, Oregon Health and Science University, for his willingness to perform a review of the work as an international expert and for sharing some of his unpublished data. The authors sincerely appreciate and honour the gifts provided by deceased donors and their families. Without their selflessness, organ donation and transplantation would not be possible.

Correspondence to: Ian Ball, Ian.Ball@lhsc.on.ca 